\title{
Using 3D printed eggs to examine the egg-rejection behaviour of wild birds
}

Branislav Igic, Valerie Nunez, Henning U. Voss, Rebecca Croston, Zachary Aidala, Analía V. López, Aimee Van Tatenhove, Mandë Holford, Matthew D. Shawkey, Mark E. Hauber

The coevolutionary relationships between brood parasites and their hosts are often studied by examining the egg rejection behaviour of host species using artificial eggs. However, the traditional methods for producing artificial eggs out of plasticine, plastic, wood, or plaster-of-Paris are laborious, imprecise, and prone to human error. As an alternative, 3D printing may reduce human error, enable more precise manipulation of egg size and shape, and provide a more accurate and replicable protocol for generating artificial stimuli than traditional methods. However, the usefulness of 3D printing technology for egg rejection research remains to be tested. Here, we applied 3D printing technology to the extensively studied egg rejection behaviour of American robins, Turdus migratorius. Eggs of the robin's brood parasites, brown-headed cowbirds, Molothrus ater, vary greatly in size and shape, but it is unknown whether host egg rejection decisions differ across this gradient of natural variation. We printed artificial eggs that encompass the natural range of shapes and sizes of cowbird eggs, painted them to resemble either robin or cowbird egg colour, and used them to artificially parasitize nests of breeding wild robins. In line with previous studies, we show that robins accept mimetically coloured and reject nonmimetically coloured artificial eggs. Although we found no evidence that subtle differences in parasitic egg size or shape affect robins' rejection decisions, 3D printing will provide an opportunity for more extensive experimentation on the potential biological or evolutionary significance of size and shape variation of foreign eggs in rejection decisions. We provide a detailed protocol for generating 3D printed eggs using either personal 3D printers or commercial printing services, and highlight additional potential future applications for this technology in the study of egg rejection. 
2 Using 3D printed eggs to examine the egg-rejection behaviour of wild birds

3

4 Branislav Igic ${ }^{1}$, Valerie Nunez ${ }^{2}$, Henning U. Voss ${ }^{3}$, Rebecca Croston ${ }^{2,4}$, Zachary Aidala ${ }^{2,5}$, Analía V.

5 López $^{6}$, Aimee Van Tatenhove ${ }^{7}$, Mande E. Holford ${ }^{8}$, Matthew D. Shawkey ${ }^{1}$, and Mark E. Hauber ${ }^{2}$

$7 \quad{ }^{1}$ Department of Biology, University of Akron, Akron, OH, USA

$8 \quad{ }^{2}$ Department of Psychology, Hunter College, and The Graduate Centre of the City University of New

9 York, NY, USA

$10{ }^{3}$ Citigroup Biomedical Imaging Centre, Weill Cornell Medical College, New York, NY, USA

$11{ }^{4}$ Department of Biology, University of Nevada - Reno, Reno, NV, USA

$12{ }^{5}$ Social and Behavioural Sciences Division, Bloomfield College, 467 Franklin Street, Bloomfield, NJ,

13 USA

$14{ }^{6}$ Department of Ecology, Genetics, and Evolution, University of Buenos Aires, Buenos Aires, 15 Argentina

$16 \quad{ }^{7}$ Department of Ecology and Evolutionary Biology, Cornell University, Ithaca, NY, USA

$17{ }^{8}$ Department of Chemistry, Hunter College, The Graduate Centre of the City University of New York, 18 and The American Museum of Natural History, NY, USA

19

20 Author for correspondence: Branislav Igic, brani.igic@gmail.com; Department of Biology, University

21 of Akron, Akron, OH 44325-3908, USA; :+1-330-396-0232 
Introduction

23 Ever since the Nobel-prize winning studies using painted eggshells by Tinbergen (1963), research on

24 avian eggs has focused on studying the biological causes and effects of variation in egg colour, size,

25 shape, and shell strength. Studies of egg rejection examine the behavioural interactions between avian

26 brood parasites and their hosts, with an aim to understand coevolutionary interactions more generally

27 (Dawkins \& Krebs 1979; Rothstein \& Robinson 1998). Brood parasitic birds, such as European

28 common cuckoos Cuculus canorus and North American brown-headed cowbirds Molothrus ater, lay

29 their eggs into nests of other species, prompting these hosts to raise parasitic offspring at the expense

30 of their own offspring (Rothstein \& Robinson 1998; Davies 2000).

In response to costly brood parasitism, many hosts have evolved the ability to identify and eliminate parasitic eggs from their nests (Davies 2000; Peer et al. 2005). Extensive experimental work using real eggs of conspecifics and parasites, as well as both real and artificial eggs painted to resemble natural or artificial colours, has revealed the abilities of host species to detect, recognize, and reject parasitic eggs in their nests (Davies \& Brooke 1989; Samas et al. 2014). The cues used by hosts to

37 discriminate own vs. parasitic eggs can include egg size, shape, colour, patterning, or a combination of

38 these traits (Rothstein 1982; Antonov et al. 2006b; Underwood \& Sealy 2006; Stoddard \& Stevens 39 2010; Stokke et al. 2010; Zölei et al. 2012). However, parasitic eggs are naturally variable in these 40 traits, and how these subtle differences, particularly in egg size and shape, influence host behaviour has 41 been difficult to test (Lahti \& Lahti 2002).

The use of real eggs in egg rejection research is invaluable but also problematic (for discussions 44 see Hauber et al. 2015; Lahti 2015). The usefulness of real eggs can be limited because the relative 45 influences of egg shape, size, and colour on rejection decisions cannot be easily differentiated 
46 (Antonov et al. 2009). The use of natural eggs in experiments also results in the destruction of viable

47 eggs, which can be problematic from ethical and management considerations. Artificial eggs are used

48 as alternatives to real eggs in experiments and are specifically useful in separating the relative

49 influences of different egg phenotypic characteristics on rejection decisions (Rothstein 1982; Hauber et

50 al. 2015; although see Lahti 2015). However, traditional artificial eggs made of plasticine, plastic,

51 wood, or plaster-of-Paris, are also of limited use (Prather et al. 2007). Their production can be

52 laborious, rely on a single mould or prototype that degenerates with extensive use, and be prone to

53 human error. For example, the mass and quality of plaster cast eggs are strongly influenced by how

54 well a plaster mixture is prepared. As a result, the final products can be constrained in quality,

55 reproducibility, and experimental validity. Indeed, artificial eggs may be rejected based on features that

56 were not experimentally manipulated or controlled (Prather et al. 2007). Finally, traditional methods

57 are not amenable to precise and fine-scale manipulation of egg shape, size, or other characteristics, and

58 thereby reduce the range of hypothesis that can be tested.

3D imaging and printing technologies may help overcome some of the limitations of traditional

61 methods for artificial egg production. 3D printers create plastic objects from digital 3D models, which

62 can be generated using photographs or mathematical formulas (e.g. Troscianko 2014). 3D printing

63 technology is advantageous over traditional methods for producing artificial eggs because it is less

64 prone to human error and allows precise and controlled manipulation of one, or several, egg traits,

65 including shape, weight, and texture. Therefore, it can both eliminate unwanted variability in mass

66 production of identical stimuli eggs and also allow precise control of how much, and in which

67 parameters, eggs vary. Unlike traditional methods, 3D printing allows the manufacture of hollow eggs,

68 which may imitate characteristics of real eggs more accurately. Lastly, digital 3D models can be easily

69 shared online for other researchers to use, enabling more replicable use of experimental stimuli eggs 
70 across different laboratories, continents, and study species, than is possible with traditional methods for

71 making artificial eggs. Despite these potential advantages, the usefulness of 3D printing technology for 72 studying egg rejection decisions remains to be tested.

We applied 3D printing technology in a pilot study to study the egg rejection behaviour of

75 American robins Turdus migratorius in relation to brood parasitism by brown-headed cowbirds.

76 Robins lay blue-green immaculate eggs, whereas cowbirds lay smaller beige eggs with spots (Croston

77 \& Hauber 2014). Previous studies using real eggs found that robins generally reject cowbird eggs

78 added to their nests (Briskie, Sealy \& Hobson 1992; Rasmussen, Sealy \& Underwood 2009). Studies

79 using plaster-cast eggs found that robins mostly accept cowbird-sized eggs if they are painted to

80 resemble robin egg colour but reject them if painted to resemble cowbird egg background colour and

81 maculation patterns (Rothstein 1982; Croston \& Hauber 2014; Kuehn, Peer \& Rothstein 2014; Lang,

82 Bollinger \& Peer 2014). However, cowbird eggs vary greatly in size and shape (18-25 mm length x 15-

$8318 \mathrm{~mm}$ breadth; Lowther 1993), and size can be an important cue for recognition of parasitic eggs by

84 hosts, along with colour (Guigueno, Sealy \& Westphal 2014). By placing plaster eggs that varied in

85 size (robin or cowbird egg size), colour (blue-green or white), and maculation pattern into the nests of

86 robins, Rothstein (1982) showed that robins use all three cues in rejection decisions, but are in general

87 more likely to reject eggs if they differ from robin eggs in at least two of these parameters. Although

88 Rothstein (1982) found that robins are more likely to reject white plaster eggs if they are the size of

89 cowbird eggs, rather than the size of robin eggs, it is still unclear whether robins' rejection decisions

90 vary along the cowbird's natural gradient of variation for egg shape and size (Mills 1987; Lowther

91 1993). For example, robins may be less likely to reject larger cowbird eggs than smaller cowbird eggs

92 because they are more similar in size to robin eggs. To test this hypothesis, we 3D printed eggs that 
93 encompass the natural variation of cowbird egg shapes and sizes to test how these characteristics 94 influence the probability of egg rejection by robins. 


\section{Materials \& Methods}

96 We performed the following steps to generate 3D printed stimuli for egg rejection research: 1) design

97 3D digital egg models, 2) 3D print artificial eggs in-house, 3) upload digital models to a commercial

98 3D printing service for mass production. We then experimentally tested the utility of 3D printed eggs

99 using American robins as models.

100

101 Design 3D digital egg models

102 We used the Blender Foundation's open-source 3D graphics software Blender v.2.70 (freely available

103 from http://www.blender.org/download/) to design 3D model representations of brown-headed cowbird

104 eggs. We designed the initial 3D model using a photograph of a brown-headed cowbird egg (accession

105 no. 13941) sourced from the publicly available digital egg collection of the Museum of Vertebrate

106 Zoology, University of California, Berkeley. To do this, we set the egg image as the background in

107 Blender, first ensuring the egg was vertical (Figure 1 D1), then created a UV Sphere (64 segments and

10832 rings), which we expanded to match the width of the cowbird egg (Figure 1 D2). We then used

109 Blender's Proportional Edit tool to distort the sphere smoothly along its vertical axis with a fall-off

110 proportional to the distance from the centre of distortion. A smaller radius of proportional editing

111 influence resulted in a more "pinched" egg shape whilst a larger radius of influence resulted in a less

112 pointed egg shape. We distorted the sphere until its shape and length matched the background egg

113 (Figure 1 D3-D4). The resulting ovoid was then resized so that its length matched that of the original

114 egg according to the scale presented in the museum photograph. To create a hollow egg that can be

115 filled with water or gel to better approximate thermodynamic properties of natural eggs (in case this

116 affected heating/cooling rates and thereby egg rejection), a hole was digitally cut at the blunt pole of

117 the egg model such that the printed egg would have a hole no larger than 1-2 mm in diameter. 
118 Although 3D printing technology may potentially provide an opportunity to make artificial eggs with

119 shells that can be punctured by hosts with beaks too small to grasp cowbird eggs (Rohwer, Spaw \&

120 Røskaft 1989), testing whether this is possible was not an aim of our study and we encourage future

121 research in this area. The egg model was then exported as a Wavefront object (*.obj) file which could

122 be imported directly into 3D printer software or uploaded to a third party website for commercial

123 printing. Note that since the completion of this field study, we have also succeeded in creating

124 printable egg models in Blender using an XYZ Math Surface where the $\mathrm{X}, \mathrm{Y}$ and $\mathrm{Z}$ values depend on

125 the parameters $\mathrm{a}, \mathrm{b}$, and $\mathrm{c}$ from the equation for an egg surface modelled by Troscianko (2014), 126 speeding up the 3D model generation process.

We also tried an alternate approach for designing digital 3D egg models. We imported the

129 cowbird egg photograph into ImageJ (freely available from imagej.nih.gov/ij) and used the multi-point

130 tool to mark 14 equidistant points on one side of the egg, pole to pole, scaling these points to match the

131 scale bar measurements provided in the egg photograph $(23.0 \mathrm{~mm}$ in length and $17.7 \mathrm{~mm}$ in breadth).

132 We saved these points as "xy coordinates" in a *.txt file, which we then converted into a Microsoft

133 Excel 2010 *.xls document and imported into Autodesk Inventor Professional 2014 (available from

$134 \mathrm{http} / / / \mathrm{www}$. autodesk.com/education/free-software/inventor-professional) as a 2D sketch. The imported

135 points were connected by control vertex-type splines and the resulting half-egg curve closed by

136 insertion of a straight line. To create a solid body from the 2D sketch, the half egg sketch was then

137 revolved one full circle around an axis defined by the previously inserted straight line. The two poles

138 of the egg were respectively too pointed or too blunt, so we manually adjusted the position of the two

139 pole end points to generate the original image's shape. The resulting sketch was saved as an *.stl file

140 and can be uploaded to websites of commercial 3D printing services (see below). 
142 We printed the 3D eggs from Acrylonitrile butadiene styrene (ABS) plastic using a MakerBot

143 Replicator 2X 3D printer. The egg object file was sliced for printing with MakerBot MakerWare v.

144 2.4.1.62 software (Figure P1). We created hollow, water filled, eggs to replicate weights and the

145 thermodynamic properties of similarly sized natural eggs. To replicate the natural variation of egg

146 shape and sizes of brown-headed cowbirds (Lowther 1993), we scaled the initial egg model to be

147 between 19-24.4 $\mathrm{mm}$ in length and 14.4-18.1 $\mathrm{mm}$ in breadth using MakerWare (final sizes were

148 measured after painting; see below). Therefore, although all eggs were designed from the same initial

149 cowbird egg, each resulting printed egg was unique in shape and size to replicate the diversity of

150 cowbird egg phenotypes and avoid potential biases of pseudo replication (Hurlbert 1984). We printed

151 eggs with four internal shell layers built on top of each other, using 0.3 and $0.2 \mathrm{~mm}$ layer thicknesses,

152 and $0 \%$ infill (Figure $1 \mathrm{P} 2$ ). We filled these eggs with water using a syringe and then sealed them

153 using thick ABS-acetone glue made by dissolving ABS in acetone to create a thick paste. We then

154 diluted this ABS-acetone glue using more acetone and coated the entire egg surface to ensure that the

155 product was watertight. Egg surfaces were sanded smooth prior to painting and between paint layers

156 when necessary. Whole eggs can also be submerged in $100 \%$ acetone (Figure 1 P3) to create a smooth 157 surface (Figure 1 P4).

158

We painted eggs blue-green and beige using two coats of nontoxic acrylic or latex house paint

160 (Behr PREMIUM PLUSTM Interior Paint), sensu Croston \& Hauber (2014). We measured the 161 reflectance of painted artificial eggs, and the background coloration of real robin and cowbird eggs, 162 following methods described in Igic et al. (2012). The background coloration of natural cowbird eggs 163 is difficult to replicate using commercial paint designed for human vision (Croston \& Hauber 2014). 164 Therefore, we used paint that provokes robins to reject artificial eggs at similar rates as they do natural 
165 cowbird eggs, and which is similar to the beige background coloration of cowbird eggs between 400-

$166700 \mathrm{~nm}$ (Figure 2b; Briskie, Sealy \& Hobson 1992; Croston \& Hauber 2014). American robins do not

167 appear to prioritise ultra-violet wavelengths over other avian-perceived wavelengths in egg rejection

168 decisions, but rather use the whole avian visible spectrum to identify foreign eggs in their nests

169 (Croston \& Hauber 2014). The effect of maculation was excluded from our study because it can be

170 used as an additional, or separate, cue to eggshell background coloration for identifying parasitic eggs

171 by robins and other species (Rothstein 1982; Stoddard \& Stevens 2010).

We measured the weight of the water-filled eggs immediately after sealing/painting and again

17412 days later to ensure that the water did not evaporate or leak; the average weight loss was $1 \%$

175 (range: $0.1-2.4 \% ; n=38$ ) with two additional eggs losing $7 \%$ and $15 \%$, respectively: these eggs were

176 discarded from the experiment. In addition to creating hollow water filled eggs, we were able to print 177 solid filled eggs using two internal shell layers with $0.3 \mathrm{~mm}$ layer thickness and $100 \%$ infill.

length $\times 18.0 \mathrm{~mm}$ breadth) and measuring their size dimensions to the nearest $0.1 \mathrm{~mm}$ using digital

181 callipers (Mitutoyo Digimatic Plastic Callipers model 700-126) and weight to the nearest $0.1 \mathrm{~g}$ using

182 an American Weigh AMW-155 (150 g x $0.1 \mathrm{~g})$ scale. We compared these measurements to those taken on plaster eggs produced following Croston \& Hauber (2014) using coefficients of variation.

\section{Upload digital models to commercial 3D printing service}

186 To provide researchers with access to 3D printed cowbird eggs without a requirement for personal 3D 187 printers or 3D modelling software, we provide our egg model designs (Files S1), allowing other 188 researchers to use our 3D designs with commercial 3D printing services. To test the utility of using 
189 commercial 3D printing services, we uploaded our designs to www.shapeways.com. We chose "white

190 strong and flexible plastic, polished" as the manufacture material, because the eggs were printed solid

191 filled and their resulting weight (3.1 g) was similar to the average weight of brown-headed cowbird 192 eggs (mean: 3.2 g, range: 2.5-3.8; Ankney \& Johnson 1985). For ease of access, our egg design can 193 also be directly printed through Shapeways (https://www.shapeways.com/shops/VN).

Experiment using $3 D$ printed eggs

196 To test the utility of 3D printed eggs, we studied the egg rejection behaviour of American robins during 197 May-July 2014, in and around Ithaca, Tompkins County, NY, USA. Robins are highly commensal with 198 humans (Vanderhoff, Sallabanks \& James 2014), and often nest on human-made structures or in 199 vegetation nearby. We located nests by searching in and around residential areas, parking lots, and 200 natural woodland areas (Croston \& Hauber 2014). Nests were deemed active if natural clutch size 201 increased between subsequent visits or if a robin was observed to be incubating the eggs. Upon finding 202 an active nest that contained two or more robin eggs, we placed a 3D printed egg that was painted blue203 green to resemble colour of robin eggs $(n=14)$ or beige to resemble the background colour of cowbird 204 eggs $(n=14)$, allocated at random. The timing of parasitism, with respect to whether the female is 205 laying or incubating eggs, does not affect probability of egg rejection in this species (Croston \& 206 Hauber 2014), so we placed eggs in nests at either of these stages. We did not remove any host eggs 207 during the experiment because removing a single egg has no effect on the rejection responses of robins 208 (Briskie, Sealy \& Hobson 1992). Every nest received an artificial egg with a unique size or shape.

We monitored all nests daily for six days following parasitism, and visually determined the 211 presence or absence of artificial eggs. Robins generally reject eggs within five days following 212 parasitism (Croston \& Hauber 2014) by grasping them in their bills and carrying them away from the 
213 nest (Video S1; Rothstein 1975; Rasmussen, Sealy \& Underwood 2009). By using a large grasp-ejector

214 species in our experiments, such as the American robin, we avoided potential spurious results of egg

215 acceptance because artificial eggs cannot be pierced (Martín-Vivaldi, Soler \& Møller 2002; Boulton \&

216 Cassey 2006; Soler et al. 2015) and isolated rejection probabilities to the recognition of parasitic eggs

217 by hosts (Hauber \& Low 2014; Mendelson 2015), rather than physical constraints of rejecting eggs of

218 particular size or shape. Indeed, our specific aim was to examine how egg size and shape influence

219 robins' egg rejection decisions, rather than the physical constraints on egg rejection. Although an ideal

220 study species would be a grasp-ejector that is known to respond to the size and shape of cowbird eggs,

221 we are unaware whether such a host has been identified. We considered rejection to have occurred

222 when the artificial egg was absent from the nest, but the nest showed no signs of partial or total

223 predation, such as missing or broken robin eggs. If the female had not been seen incubating for the past

224 three days and the eggs were cold, we considered the artificial egg to have been rejected via nest

225 abandonment. A single nest was abandoned following experimental parasitism with a beige egg,

226 whereas no nests were abandoned following experimental parasitism with a blue-green egg. We

227 considered acceptance to have occurred if the artificial egg was still present in the nest after six days

228 following experimental parasitism. We excluded any experimental nests from our analysis where all

229 the robin's eggs hatched prior to this six day acceptance period and the artificial egg was still present in

230 the nest. For nests that reached an experimental outcome (either rejection or acceptance of artificial

231 eggs) before eggs began to hatch, we parasitized those nests again with an artificial egg of the alternate

232 colour immediately after determining acceptance or rejection of the previous egg. This was done to

233 maximise the power of our analyses through a repeated-measures experimental design. In this way, we

234 successfully completed experiments at six nests with two artificial eggs in succession and 16 nests with

235 a single artificial egg. In addition, five nests were predated following parasitism and five nests had

236 eggs that hatched before an experimental outcome was reached, and were excluded from the analyses. 
239 female was flushed off the nest during parasitism, and the order in which artificial eggs were presented.

240 Prior work showed no effect of presentation order on the outcome of egg colour experiments in this

241 population (Croston \& Hauber 2014); nevertheless, we still tested for an order effect statistically (see 242 below).

Handling of wild birds, including nests and eggs, were carried out in accordance with Federal and State guidelines and laws. Work conducted on private properties was with consent of the affected landowners, and approved by the Institutional Animal Care and Use Committee of Hunter College, City University of New York (No. MH 2/16-T3) and US bird banding laboratory (no. 23681).

Statistical analysis

250 We used penalized-likelihood models to test whether rejection of artificial eggs was affected by their 251 colour, size, shape, order of presentation, or whether the female was flushed off the nest when eggs were added. To examine whether artificial egg shape and size affected rejection responses, we first used principal component analysis on egg length and breadth measurements to produce two orthogonal axes: PC1 explained $99 \%$ of the total variance and compared average egg length (loading: -0.83 ) and breadth (loading: -0.55), and therefore was a measure of total egg size (Figure 3c); PC2 explained $1 \%$

256 of the total variance and compared egg length (loading: 0.55) to egg breadth (loading: -0.83 ), and 257 therefore was a measure of egg shape (Figure 3c). There was a complete separation in the response outcomes of our data in regards to egg colour (all blue-green eggs were accepted); therefore, we used 259 Firth's penalized-likelihood logistic regression for all tests and tested each factor separately while also 260 controlling for nest ID. Not only is penalized-likelihood logistic regression useful in overcoming 
261 problems of complete separation, but it outperforms traditional logistic regression when sample sizes

262 are small to medium (Heinze \& Schemper 2002). We used rejection response (binary outcome:

263 accept/reject) as an independent variable and fit each factor (colour, egg size, egg shape, etc.) as

264 explanatory variables. Removing a nest that was abandoned following experimental parasitism with a

265 beige egg did not affect our results (Soler et al. 2015). We tested whether flushing females off of their

266 nest during simulated parasitism affected their probability of rejecting beige eggs using a Fisher's exact

267 test (Hanley et al. 2015). We present mean odds ratios and $95 \%$ confidence intervals for all tests.

268

In addition to testing the effects of egg colour, we tested whether rejection of artificial eggs by

270 robins is influenced by whether eggs are made using 3D printing or plaster-of-Paris. Here we compared

271 our rejection data of 3D printed eggs to rejection data of a recent study that investigated egg rejection

272 behaviour using the same robin population and paint colours, but using $21.4 \mathrm{~mm}$ length $\mathrm{x} 16.4 \mathrm{~mm}$

273 breadth eggs made of plaster (Croston \& Hauber 2014). This included robins' responses to 13 blue-

274 green plaster eggs and 19 beige plaster eggs. We fit a penalized-likelihood logistic regression with egg

275 rejection response as the independent variable; nest ID, egg colour, egg make, and the interaction

276 between egg colour and make as fixed effects. The interaction was not significant $\left(\chi_{1}^{2}=0.46, P=0.5\right.$ ),

277 so we refit the model without this interaction. All penalized-likelihood logistic regressions were fit

278 using the logistf package (Ploner et al. 2013), and all statistical tests were two-tailed $(\alpha=0.05)$ and

279 conducted using R v 3.0.1 (Team 2013). 


\section{Results}

281 American robins accepted all eggs painted to resemble robin egg colour and rejected $79 \%$ of eggs 282 painted to resemble cowbird egg colour (Video S1), but we detected no effects of egg size or shape on 283 rejection probability (Figure 2; 3). Robins were more likely to reject beige eggs than blue-green eggs 284 (beige vs. blue-green: odds ratio $=15.0,95 \%$ CI $[1.3,425.7] ; \chi_{1}^{2}=4.79, P=0.029 ;$ Figure $2 \mathrm{c}$ ). Egg 285 size $\left(\mathrm{PC} 1:\right.$ odds ratio $=1.5,95 \% \mathrm{CI}[0.8,3.1] ; \chi_{1}^{2}=1.5, P=0.22$; Figure $\left.3 \mathrm{a}\right)$, egg shape (PC2: odds 286 ratio $=1159.5,95 \% \mathrm{CI}[0.0001,>10000] ; \chi_{1}^{2}=0.72, P=0.40 ;$ Figure $\left.3 \mathrm{a}\right)$, the order in which eggs 287 were presented ( $1^{\text {st }}$ vs. $2^{\text {nd. }}$ odds ratio $=3.1,95 \% \mathrm{CI}[0.4,36.4] ; \chi_{1}^{2}=1.10, P=0.30$; Figure $\left.3 \mathrm{~b}\right)$, and 288 whether the female was flushed off the nest when the artificial egg was added (no vs. yes: odds ratio = $2890.1,95 \%$ CI $[0.001,2.2] ; \chi_{1}^{2}=1.92, P=0.17$; Figure $3 \mathrm{c}$ ) did not influence rejection probability in our 290 study. When considering beige eggs alone, size (PC1: odds ratio $=1.2 ; 95 \%$ CI $[0.6,3.1] ; \chi_{1}^{2}=0.15, P$ $291=0.7$; Figure $3 a)$ and shape $\left(\mathrm{PC} 2\right.$ : odds ratio $=6.1 ; 95 \% \mathrm{CI}[0.04,848.1] ; \chi_{1}^{2}=0.56, P=0.45 ;$ Figure $2923 \mathrm{a}$ ), and whether the female was flushed (odds ratio $=0 ; 95 \%$ CI $[0,10.4]$; Fisher's exact test $P>$ 293 0.99) did not influence their probability of being rejected.

Next, we compared our data on the rejection of variably sized 3D printed eggs to data from a 296 recent study that examined egg rejection behaviour of the same robin population using the same paint 297 colours, but invariant artificial eggs made of plaster (Croston \& Hauber 2014). Egg colour (beige vs. 298 blue-green: odds ratio $=14.2 ; 95 \%$ CI $[1.6,291.5] ; \chi_{1}^{2}=5.9, P=0.02$; Figure $\left.3 \mathrm{~d}\right)$, but not manufacture 299 type (plaster cast vs. 3D printed: odds ratio $=1 ; 95 \%$ CI $[0.4,2.5] ; \chi_{1}^{2}<0.01, P>0.99$; Figure 3d), 300 was a significant predictor of rejection response. 
302 The size dimensions and weight of eggs printed the same size were low in variance, and lower 303 than that of same sized plaster-cast eggs (Table 1). In particular, 3D printing was much better at 304 producing artificial eggs with a consistent weight (Table 1), perhaps because the weight of plaster eggs 305 is strongly dependent on how the plaster mixture is prepared. 3D printing was also more precise in 306 reproducing a set size and shape of eggs than the plaster method (Table 1). 


\section{Discussion}

308 Here, we demonstrate the utility of 3D printing technology in egg rejection studies. We provide a

309 detailed protocol to enable other researchers to use the economical and flexible 3D design and printing

310 technologies in their own research. Our experimental findings correspond with previous studies

311 showing that colour is as an important cue for rejection of cowbird eggs by robins (Rothstein 1982;

312 Croston \& Hauber 2014; although see Lorenzana, Sealy \& Murphy 2002). Although previous studies

313 showed that robins are more likely to reject eggs smaller than their own (Rothstein 1982), our results

314 suggest that egg rejection decisions of robins do not vary across the natural variation of cowbird egg

315 shapes and sizes. However, our sample sizes were limited and a more extensive experiment is needed

316 to detect weak effects. The lack of statistical effects of parasitic egg shape and size are consistent with

317 robins rejecting spherical and elongate egg shapes from their nests at similar rates (Underwood \&

318 Sealy 2006) and cowbirds parasitizing host species' nests indiscriminately with respect to host egg size

319 (Mills 1987). The precise and controlled manipulation of stimuli characteristics using 3D printing

320 technology showcased here will enable others to expand upon our findings and test similar recognition

321 mechanisms in other host species.

3D printing permits precise, reproducible, and accurate manipulation of stimuli characteristics

324 at a level that cannot be achieved by traditional methods for making artificial eggs. However, 3D

325 printed eggs may not necessarily produce more biologically meaningful results than traditional

326 artificial eggs, but rather expand the range of hypotheses that can be tested. 3D printing enables

327 researchers to apply a more standardized and replicable technique in production of artificial eggs as

328 evidenced by lower variability when producing identical stimuli, and enables much finer control over

329 how much and in which characteristics stimuli vary. Furthermore, in contrast to traditional methods,

330 3D printing allows the replicable use of experimental stimuli across studies because digital 3D egg 
331 models can be exchanged between researchers. For example, we provide our design schematics of

332 cowbird eggs (File 1) that can be used by others in combination with personal or commercial 3D

333 printers. Commercial 3D printing services also allow manipulation of characteristics of uploaded 3D

334 image files, and thereby allow researchers to design egg stimuli for their specific needs without the

335 need of a personal 3D printer. We conducted proof of concept experiments using these commercially

336 produced 3D printed cowbird eggs and found that they can also be accepted when painted to resemble

337 robin egg colour $(n=2)$ and rejected when painted to resemble cowbird egg background colour $(n=1)$.

338 Although 3D printing does not necessarily overcome the potential limitations of using artificial stimuli

339 in behavioural research (Lahti 2015), it is an important step towards creating more natural-like artificial

340 stimuli to reduce potential biases.

Beyond the uses demonstrated here, 3D printing has the potential for widespread applicability

across many areas of behavioural ecology, comparative psychology, and neuroethology. For brood

parasitism research, 3D printing may enable more precise examination of the sensory and motor controls of, and their constraints on, egg recognition and rejection (Rothstein 1982; Antonov et al.

346 2009). It allows precise manipulation of egg shape and potentially, shell thickness, making it

347 potentially possible to create egg shapes that can, or cannot, be grasped and removed from the nest

348 (Rohwer \& Spaw 1988) or eggs with shells that are thin enough, or too thick, for hosts to reject by

349 puncturing the shell (Antonov et al. 2006a; Spottiswoode 2010). Indeed, when artificial eggs cannot be

350 pierced by small hosts, rejection of eggs by piercing their shells may not be possible, and thereby

351 generate spurious results of egg acceptance or nest abandonment, instead of egg ejection

352 (Martín-Vivaldi, Soler \& Møller 2002; Boulton \& Cassey 2006; Soler et al. 2015). However, the

353 capacity for 3D printing to produce artificial eggs that can be rejected by hosts via puncturing their

354 shells may be possible in the future, with the advent of light and brittle printable materials; this concept 
355 remains to be tested. Weight to size ratio and surface texture can also be altered to test how these

356 properties affect egg rejection behaviour. More broadly, 3D printing is likely to revolutionize

357 behavioural research by allowing precise manipulation and economical production of controlled 358 stimuli. Potential future applications include, but are not limited to, studies of visual recognition of 359 predators, prey, and conspecifics (Nelson 2010); sexually selected visual signals (Callander et al. 360 2013); and the evolution of egg morphology (Preston 1953). 
Acknowledgements

362

363

364

365

366

367

368

369

370

371

372

373

374

375

376

377

378

379

380

381

382

383

384

385

386

387

388

389

390

391

392

393

394

395

396

397

398

399

400

401
We thank Cornell University, Ithaca College, and the many landowners that allowed us to conduct our research on their property. We thank the Shawkey lab for helpful comments.

\section{References}

Ankney CD, Johnson SL. 1985. Variation in weight and composition of brown-headed cowbird eggs. The Condor 87:296-299.

Antonov A, Stokke BG, Moksnes A, Kleven O, Honza M, Røskaft E. 2006a. Eggshell strength of an obligate brood parasite: a test of the puncture resistance hypothesis. Behavioural Ecology and Sociobiology 60:11-18.

Antonov A, Stokke BG, Moksnes A, Røskaft E. 2009. Evidence for egg discrimination preceding failed rejection attempts in a small cuckoo host. Biology Letters 5:169-171.

Antonov A, Stokke BG, Moksnes A, Røskaft E, Brittingham M. 2006b. Egg rejection in marsh warblers (Acrocephalus palustris) heavily parasitized by common cuckoos (Cuculus canorus). The Auk 123:419-430.

Boulton RL, Cassey P. 2006. An inexpensive method for identifying predators of passerine nests using tethered artificial eggs. New Zealand Journal of Ecology 30:377-385.

Briskie JV, Sealy SG, Hobson KA. 1992. Behavioral defenses against avian brood parasitism in sympatric and allopatric host populations. Evolution 46:334-340.

Callander S, Kahn AT, Maricic T, Jennions MD, Backwell PR. 2013. Weapons or mating signals? Claw shape and mate choice in a fiddler crab. Behavioural Ecology and Sociobiology 67:11631167.

Croston R, Hauber ME. 2014. Spectral tuning and perceptual differences do not explain the rejection of brood parasitic eggs by American robins (Turdus migratorius). Behavioural Ecology and Sociobiology 68:351-362.

Davies NB. 2000. Cuckoos, cowbirds and other cheats. London: T. \& A.D. Poyser.

Davies NB, Brooke MDL. 1989. An experimental study of co-evolution between the cuckoo, cuculus canorus, and its hosts. I. host egg discrimination. Journal of Animal Ecology 58:207-224.

Dawkins R, Krebs JR. 1979. Arms races between and within species. Proceedings of the Royal Society B: Biological Sciences 205:489-511.

Guigueno MF, Sealy SG, Westphal AM. 2014. Rejection of parasitic eggs in passerine hosts: size matters more for a non-ejecter. The Auk 131:583-594.

Hanley D, Samas P, Heryan J, Hauber ME, Grim T. 2015. Now you see it, now you don't: flushing hosts prior to experimentation can predict their responses to brood parasitism. Scientific Reports 5.

Hauber M, Low J. 2014. Ch. 9: Avian brood parasitism: how to spot a foreign egg in the nest? In: Yasukawa K, ed. Animal Behavior, Volume 3 Integration and Application with Case Studies. Santa Barbara: Praeger Press.

Hauber ME, Tong L, Bán M, Croston R, Grim T, Waterhouse GIN, Shawkey MD, Barron AB, Moskát C. 2015. The value of artificial stimuli in behavioral research: making the case for egg rejection studies in avian brood parasitism. Ethology doi: 10.1111/eth.12359 
Heinze G, Schemper M. 2002. A solution to the problem of separation in logistic regression. Statistics in medicine 21:2409-2419.

Hurlbert SH. 1984. Pseudoreplication and the design of ecological field experiments. Ecological Monographs 54:187-211.

Igic B, Cassey P, Grim T, Greenwood DR, Moskát C, Rutila J, Hauber ME. 2012. A shared chemical basis of avian host-parasite egg colour mimicry. Proceedings of the Royal Society B: Biological Sciences 279:1068-1076.

Kuehn MJ, Peer BD, Rothstein SI. 2014. Variation in host response to brood parasitism reflects evolutionary differences and not phenotypic plasticity. Animal Behaviour 88:21-28.

Lahti DC. 2015. The limits of artificial stimuli in behavioral research: the umwelt gamble. Ethology doi: $10.1111 /$ eth. 12361

Lahti DC, Lahti AR. 2002. How precise is egg discrimination in weaverbirds? Animal Behaviour 63:1135-1142.

Lang AK, Bollinger EK, Peer BD. 2014. Effect of parasite-to-host egg ratio on egg rejection by a brown-headed cowbird host. The Auk 131:694-701.

Lorenzana JC, Sealy SG, Murphy M. 2002. Did blue eggs of black-billed (Coccyzus erythropthalmus) and yellow-billed (C. americanus) cuckoos evolve to counter host discrimination? The Auk 119:851-854.

Lowther PE. 1993. Brown-headed cowbird (Molothrus ater). In: Poole A, editor. The birds of North America online: Cornell Lab of Ornithology, Ithaca.

Martín-Vivaldi M, Soler M, Møller AP. 2002. Unrealistically high costs of rejecting artificial model eggs in cuckoo Cuculus canorus hosts. Journal of Avian Biology 33:295-301.

Mendelson TC. 2015. Distinguishing perceptual and conceptual levels of recognition at group boundaries. Evolutionary Ecology 29:205-215.

Mills AM. 1987. Size of host egg and egg size in brown-headed cowbirds. The Wilson Bulletin 99:490491.

Nelson XJ. 2010. Visual cues used by ant-like jumping spiders to distinguish conspecifics from their models. Journal of Arachnology 38:27-34.

Peer BD, Rothstein SI, Kuehn MJ, Fleischer RC. 2005. Host defenses against cowbird (Molothrus spp.) parasitism: implications for cowbird management. Ornithological Monographs 2005:8497.

Ploner M, Dunkler D, Southworth H, Heinze G. 2013. logistf: Firth's bias reduced logistic regression. R package version 1.21 Available at http://CRAN.R-project.org/package=logistf (accessed 8 January 2015).

Prather JW, Cruz A, Weaver PF, Wiley JW. 2007. Effects of experimental egg composition on rejection by village weavers (Ploceus cucullatus). The Wilson Journal of Ornithology 119:703711.

Preston F. 1953. The shapes of birds' eggs. The Auk 70:160-182.

Rasmussen JL, Sealy SG, Underwood TJ. 2009. Video recording reveals the method of ejection of brown-headed cowbird eggs and no cost in American robins and gray catbirds. The Condor 111:570-574.

Rohwer S, Spaw CD. 1988. Evolutionary lag versus bill-size constraints: a comparative study of the acceptance of cowbird eggs by old hosts. Evolutionary Ecology 2:27-36.

Rohwer S, Spaw CD, Røskaft E. 1989. Costs to northern orioles of puncture-ejecting parasitic cowbird eggs from their nests. The Auk:734-738.

Rothstein SI. 1975. Evolutionary rates and host defenses against avian brood parasitism. American Naturalist 109:161-176. 
449 Rothstein SI. 1982. Mechanisms of avian egg recognition: which egg parameters elicit responses by $450 \quad$ rejecter species? Behavioural Ecology and Sociobiology 11:229-239.

451 Rothstein SI, Robinson SK. 1998. Parasitic birds and their hosts. NewYork: Oxford University Press.

452 Samas P, Hauber ME, Cassey P, Grim T. 2014. Host responses to interspecific brood parasitism: a byproduct of adaptations to conspecific parasitism. Frontiers in Zoology 11:34.

Soler M, Ruíz-Raya F, Roncalli G, Ibanez Á., Juan D. 2015. Nest desertion cannot be considered an egg-rejection mechanism in a medium-sized host: an experimental study with the common blackbird (Turdus merula). Journal of Avian Biology doi: 10.1111/jav.00571

Spottiswoode C. 2010. The evolution of host-specific variation in cuckoo eggshell strength. Journal of Evolutionary Biology 23:1792-1799.

Stoddard MC, Stevens M. 2010. Pattern mimicry of host eggs by the Common Cuckoo, as seen through a bird's eye. Proceedings of the Royal Society B: Biological Sciences 277:1387-1393.

Stokke BG, Polaciková L, Dyrcz A, Hafstad I, Moksnes A, Røskaft E. 2010. Responses of reed warblers Acrocephalus scirpaceus to non-mimetic eggs of different sizes in a nest parasitism experiment. Acta Ornithologica 45:98-104.

Team RDC. 2013. R: a language and environment for statistical computing. v3.0.1 ed. Vienna, Austria: R Foundation for Statistical Computing.

Tinbergen N. 1963. On aims and methods of ethology. Zeitschrift für Tierpsychologie 20:410-433.

Troscianko J. 2014. A simple tool for calculating egg shape, volume and surface area from digital images. Ibis 156:874-878.

Underwood TJ, Sealy SG. 2006. Influence of shape on egg discrimination in American robins and gray catbirds. Ethology 112:164-173.

Vanderhoff N, Sallabanks R, James FC. 2014. American Robin (Turdus migratorius). In: Poole A, editor. The birds of North America online: Cornell Lab of Ornithology, Ithaca.

Zölei A, Hauber ME, Geltsch N, Moskát C. 2012. Asymmetrical signal content of egg shape as predictor of egg rejection by great reed warblers, hosts of the common cuckoo. Behaviour 149:391-406. 


\section{Figure legends:}

480 Figure 1: Protocol for producing 3D printed replicas of cowbird eggs. First, a 3D digital egg model was 481 designed from a photograph of a real cowbird egg using Blender software (D1-D4). These digital 482 models were then printed using either a MakerBot Replicator 2X 3D printer (P1-P4) or commercial 483 web-based 3D printing services. The resulting eggs were then painted blue-green or beige. Scale bar: 1 $484 \mathrm{~cm}$.

485

486 Figure 2: Summary of artificial egg colours used in experiments and their rate of rejection by robins.

487 (a) Photographs of natural American robin nests containing an introduced cowbird-sized blue-green 488 (top) or beige (bottom) painted 3D printed egg. (b) Average reflectance of natural robin eggs, natural 489 cowbird eggs, and 3D printed eggs painted blue-green or beige to respectively resemble robin or 490 cowbird egg colours. (c) Proportion of blue-green or beige painted eggs (of all sizes) that were rejected 491 by robins. Numbers above bars illustrate sample sizes.

493 Figure 3: Factors that did not influence rejection of artificial eggs by robins in our experiments. (a) 494 Acceptance and rejection outcomes for 3D printed eggs in relation to their size and shape. Brown495 headed cowbird eggs vary between 18-25 mm in length and 15-18 $\mathrm{mm}$ in breadth (Lowther 1993). 496 Grey dotted lines represent the directions of principal component axes. Proportion of eggs rejected by 497 robins in relation to (b) the order of presentation; (c) whether the female was flushed off the nest 498 during parasitism; (d) both colour and make of artificial eggs (plaster vs. 3D printed), with plaster egg 499 data sourced from Croston \& Hauber (2014). Numbers above bars illustrate sample sizes. 
1

Protocol for producing 3D printed replicas of cowbird eggs.

First, a 3D digital egg model was designed from a photograph of a real cowbird egg using Blender software (D1-D4). These digital models were then printed using either a MakerBot Replicator 2X 3D printer (P1-P4) or commercial web-based 3D printing services. The resulting eggs were then painted blue-green or beige. Scale bar: $1 \mathrm{~cm}$. 


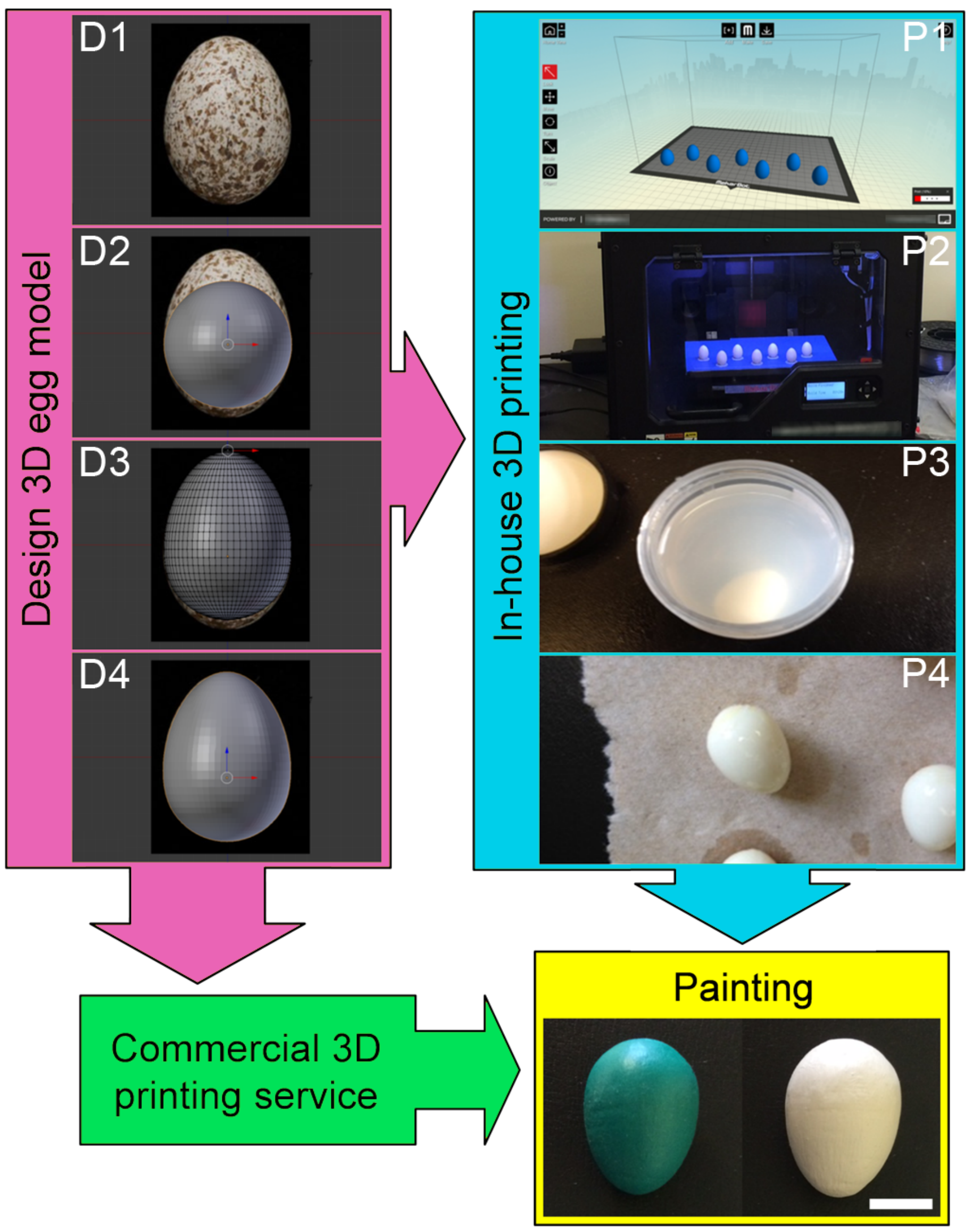




\section{2}

Summary of artificial egg colours used in experiments and their rate of rejection by robins.

(a) Photographs of natural American robin nests containing an introduced cowbird-sized bluegreen (top) or beige (bottom) painted 3D printed egg. (b) Average reflectance of natural robin eggs, natural cowbird eggs, and 3D printed eggs painted blue-green or beige to respectively resemble robin or cowbird egg colours. (c) Proportion of blue-green or beige painted eggs (of all sizes) that were rejected by robins. Numbers above bars illustrate sample sizes.
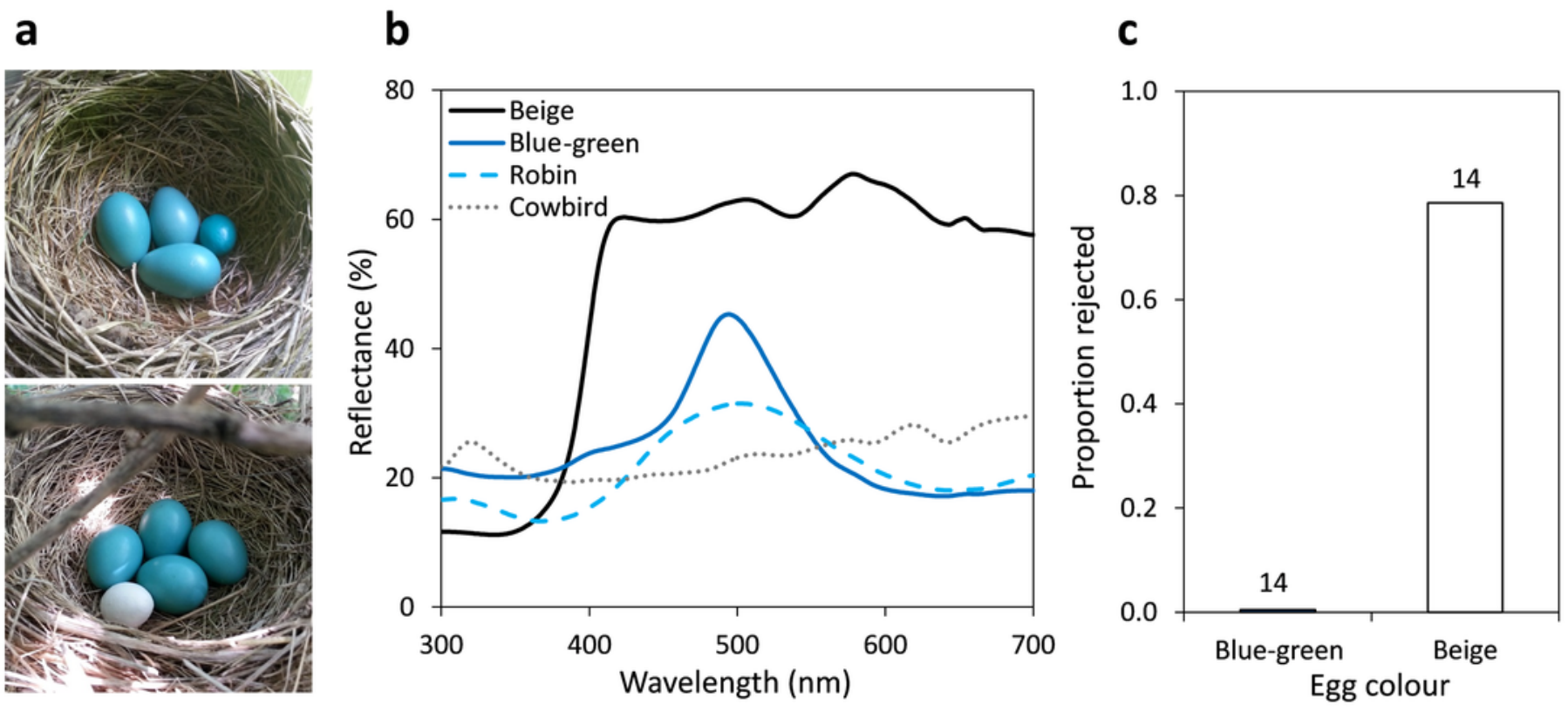


\section{3}

Factors that did not influence rejection of artificial eggs by robins in our experiments.

(a) Acceptance and rejection outcomes for 3D printed eggs in relation to their size and shape. Brown-headed cowbird eggs vary between $18-25 \mathrm{~mm}$ in length and $15-18 \mathrm{~mm}$ in breadth (Lowther 1993). Grey dotted lines represent the directions of principal component axes. Proportion of eggs rejected by robins in relation to (b) the order of presentation; (c) whether the female was flushed off the nest during parasitism; (d) both colour and make of artificial eggs (plaster vs. 3D printed), with plaster egg data sourced from Croston \& Hauber (2014). Numbers above bars illustrate sample sizes.
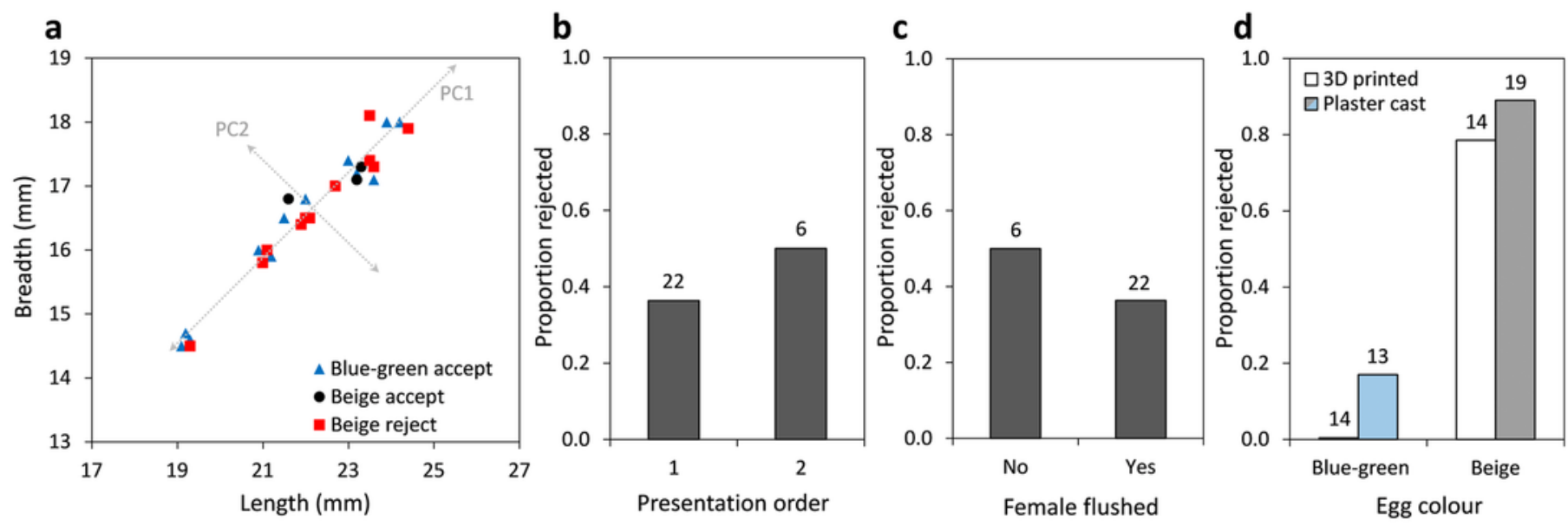
Table $\mathbf{1}$ (on next page)

Coefficients of variation expressed as percentages for size and weight of 3D printed and plaster-cast artificial eggs. 


\begin{tabular}{lccccc}
\hline & $n$ & Length & Breadth & $\begin{array}{c}\text { Aspect } \\
\text { ratio }\end{array}$ & Weight \\
\hline 3D printed & 80 & $0.54 \%$ & $0.73 \%$ & $0.82 \%$ & $0.90 \%$ \\
Plaster-cast & 63 & $2.34 \%$ & $0.28 \%$ & $2.39 \%$ & $15.00 \%$ \\
\hline
\end{tabular}

3

4 